\title{
Microscopy of Oxide Nanoparticles for Energy Applications
}

\author{
Joysurya Basu, Sanjit Bhowmick, Jonathan P. Winterstein, Chenchen Wang, Yuan Xue \\ and C. Barry Carter
}

Chemical, Materials and Biomolecular Engineering Dept., University of Connecticut, 191 Auditorium Road Unit 3222, Storrs, CT 06269-3222

The search of clean alternative energy involves catalytic reforming of high-energy-density liquid fuels and conversion of environmentally toxic byproducts to relatively benign compounds. Conductivity of the catalyst may be an added plus in order to separate out the fuel. Ceria and its derivatives, in the pure and doped form, might become useful material for such catalytic reforming and conversion processes $[1,2] . \mathrm{BaCeO}_{3}$, though structurally very different, can be seen as a derivative of ceria as Ba-doped ceria is chemically very similar to the $\mathrm{BaCeO}_{3}$. $\mathrm{Ba}$-doped ceria is also expected to be a potential material for catalytic reforming and conversion. Protonic conductivity for hydrogen separation might be introduced in this material by doping [3]. Catalytic reforming and conversion efficiencies strongly depend on the chemistry and surface activity of the material. In this light nanostructured materials may be more efficient than the conventional material of similar chemistry. Recently a cost-effective and chemically easy technique has been developed to synthesize pure-and doped-ceria and its derivatives [4-6]. In this paper the structure, growth mechanism and the chemistry of the particles will be reported.

Dilute Aqueous solution of cerium nitrate hexahydrate was reacted with a dilute solution of similar strength of hexamethylene tetramine at $80^{\circ} \mathrm{C}$ temperature to synthesize the pure ceria nanoparticles. In order to dope the particles with $\mathrm{La}$ and $\mathrm{Gd}$, the aqueous solutions of the nitrate salts of the metals were mixed in the reacting solution. Similarly for the synthesis of pure- and doped- $\mathrm{BaCeO}_{3}$, dilute aqueous solutions of the nitrate salts of the metals were reacted with an aqueous solution of hexamethylene tetramine at $80^{\circ} \mathrm{C}$. The particles have been characterized by TEM.

The pure- $\mathrm{CeO}_{2}$ particles are cuboidal in shape and 10-25 $\mathrm{nm}$ in size (Fig. 1). High-resolution microscopy and image simulation indicates that the particles grow by ionic attachments in aqueous medium and atomic level steps are present on the surfaces. La-doping does not change the particle morphology considerably whereas the particles are of irregular shape after Gd-doping (Fig. 2). However, dynamic equilibrium between 111 and 200 surfaces are observed under high-resolution imaging condition. It is understood that surface energies of the 111 and 200 facets determine the particle shapes. EELS studies indicate that the dopants are distributed homogeneously in the particles and $\mathrm{Ce}$ is predominantly in the +4 state with some +3 ions present.

Ba-doped ceria or the $\mathrm{BaCeO}_{3}$ particles are also cuboidal in shape and they are 10-25 $\mathrm{nm}$ in size. $\mathrm{Zr}$ and Co doipng results in a reduced particle size by about 5-10 nm. Zr-doping also changes the particle morphology (Fig. 3 \& 4). In these particles also 111 and 200 facets can be observed. Lowtemperature synthesis of several other oxide nanoparticles (e.g. $\mathrm{La}_{1-\mathrm{x}} \mathrm{Sr}_{\mathrm{x}} \mathrm{MnO}_{3}$ ) is being carried out. Microscopic investigation of their growth mechanism is underway.

It can be concluded from this study that it is possible to synthesize pure- and doped-nanoparticles of ceria and its derivatives by a simple low-temperature hydrothermal synthesis technique. To the best of the knowledge of the authors, it is one of the very early works on the low-temperature 
synthesis of ceria and its derivatives [7]. The particles grow by ionic attachments and surface energy plays an important role in the shape evolution of the particles.

[1] C.B. Carter and M.G. Norton: Ceramic Materials: Science and Engineering, Springer, New York, 2007.

[2] S.R. Gilliss, J. Bentley, C.B. Carter, Appl. Surf. Sci., 241 (2005) 61.

[3] A. Suresh, J. Basu, N.M. Sammes, C. B. Carter, B. A. Wilhite (2009) (submitted).

[4] J. Basu, J. P. Winterstein, S. Bhowmick, C. B. Carter, (2009) Submitted.

[5] D. S. Zhang, L. Huang, J. P. Zhang, L. Y. Shi, J. Mater. Sci., 43 (2008) 5647.

[6] M. Yoshimura, K. Byrappa, J. Mater. Sci., 43 (2008) 2085.

[7] R. Divakar, J. Basu, C.B. Carter, Micro. Microanal. 13 (2007) 980CD.

[8] The authors acknowledge the support from the University of Connecticut. JPW acknowledges support from the Air Force in the form of a NDSEG Fellowship.

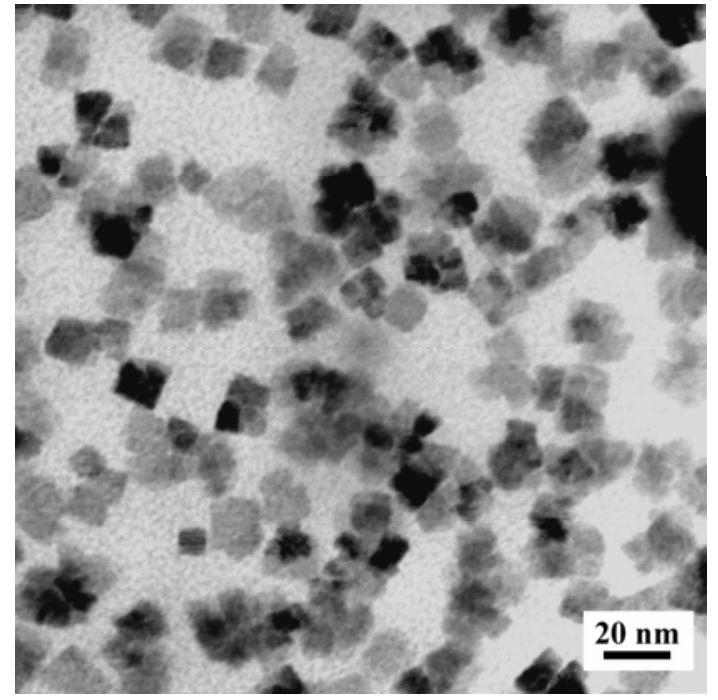

Figure 1: Hydrothermally synthesized pure$\mathrm{CeO}_{2}$ nanoparticles. Particles are cuboidal in shape.

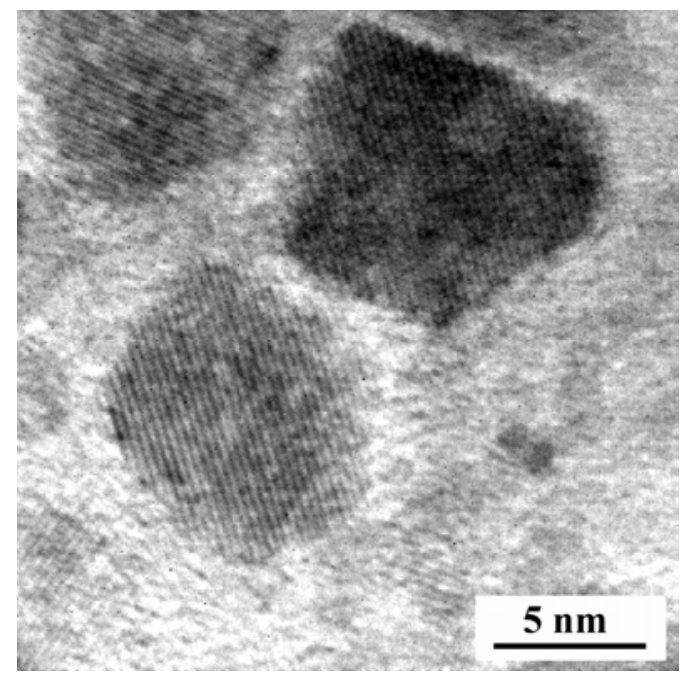

Figure 3: HRTEM image of Zr-doped $\mathrm{BaCeO}_{3}$. Particles are not cuboidal.

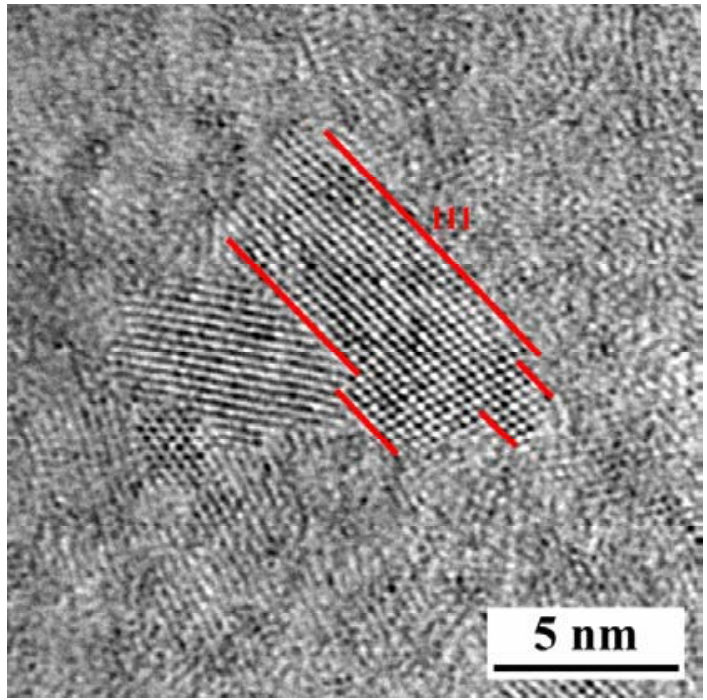

Figure 2: HRTEM image of Gd-doped $\mathrm{CeO}_{2}$. Particles are not cuboidal and 111 faceting can be seen.

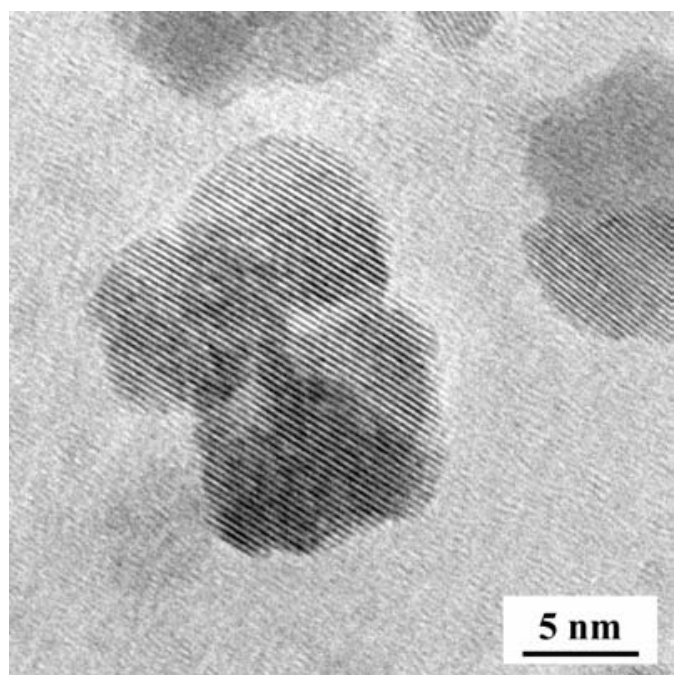

Figure 4: HRTEM image of Co-doped $\mathrm{BaCeO}_{3}$. Faceting is not so predominant. 